\title{
LES GRANDS ENSEMBLES COMME PAYSAGE
}

Paru dans "Cahiers de la Méditerranée", n 60 : "Paysages urbains (XVIe-XXe Siècles)", CMCC (Centre méditerranéen de Culture contemporaine, Université de Nice Sophia Antipolis), Tome II, Nice, Juin 2000, pp.157-178.

\section{Les architectes et le paysage, en guise d'introduction}

Le concept de paysage est d'utilisation récente pour les architectes, et plus généralement pour la ville. Le paysage est en effet plus volontiers ce qui, en se référant à la nature, représente l'espace rural et donc s'oppose à l'espace urbain. On convient aujourd'hui que le paysage est d'abord un regard, une construction intellectuelle de peintre, d'écrivain et de savant, avant d'être l'état d'une étendue aménagée et de devenir l'objet d'un métier agissant sur un espace dominé par le végétal.

L'architecture, art symbolique comme le qualifie Hegel, est, comme art, l'un des plus éloignés de la nature. Ses formes matérielles, les constructions, sont plus volontiers minérales que végétales, et elles n'ont cessé de se dissocier de la nature où elles puisent cependant leurs matériaux-ressources. L'histoire du chapiteau est ainsi celle d'un élément qui tente de faire oublier sa référence à la nature.

L'architecte n'a donc été qu'accessoirement mêlé à l'histoire du paysage, sauf lorsqu'il s'est intéressé à la place que pouvait retrouver la "nature" dans la ville, et en "paysageant" son architecture, en particulier par le jardin. La cité-jardin de l'anglais E. Howard, que dessine l'architecte R. Unwin 1 , est, dans ce sens et au début de ce siècle, une tentative de surmonter cette opposition et peut-être une tentative de faire une "ville-paysage". À la même époque en France, l'embellissement de la ville est au programme de la première loi d'urbanisme française, la loi Cornudet de 1919, qui n'envisage l'extension et l'aménagement pour laquelle elle a été adoptée qu'accompagné de cet embellissement. L'habitat y occupe une place essentielle, comme la circulation, mais aussi la pratique nouvelle du sport associant le stade et le jardin.

L'architecte a longtemps perçu la ville comme l'addition des formes particulières que sont les constructions ${ }^{2}$. Chaque construction a une forme qualifiable esthétiquement, et souvent et de plus en plus, comme le dit l'architecte B. Huet ${ }^{3}$, "l"architecture [a joué] contre la ville". Le bâtiment à construire doit surpasser le précédent (et cela peut être aussi la visée de son commanditaire). La chose est valable à Barcelone comme à New York.

La ville, somme de constructions, est donc souvent devenue pour l'architecte un ensemble d'occasions parcellaires de se distinguer.

Les circonstances où l'idée de la ville dans son ensemble subsume l'édifice particulier surviennent lorsque la ville est (s'offre à être) édifiée comme une oeuvre totalement neuve, comme ville nouvelle (Palmanova, Vitry le François à la Renaissance, Chandigarh en ce siècle...). Ce type de ville est presque "contre nature", tellement il ignore le

\footnotetext{
1. Il concrétise le projet en s'inspirant de l'autrichien C. Sitte qui analysait la ville comme un paysage et inaugurait ainsi le pittoresque.

2. Sauf peut-être Alberti qui concevait la ville comme une grande maison, et à sa suite le pape Sixte Quint, qui fait la synthèse de la Rome baroque, la met en perspective pour que le fidèle perçoive la gobalité de la ville sainte, éternelle

3. Huet (Bernard), "L'architecture contre la ville", in AMC ni 14, Paris, déc. 1986, p. 10-13.
} 
"génie du lieu", pour lui préférer la géométrie du cosmos, l'harmonie divine ou la summétria démocratique ${ }^{4}$.

L'unité de la ville, ou de ses quartiers, comme association d'édifices homogènes, est pourtant bien une réalité. Elle se fait lorsque la "règle", pour reprendre les catégories de F. Choay, prend le pas sur le "modèle", lorsque le pragmatisme l'emporte sur l'utopie, l'imitation ou plutôt la convention (et le type qui y correspond) a raison de la fièvre d'invention et d'innovation.

Précisément le concept de paysage me semble apparaître chez les architectes et les urbanistes lorsque l'intérêt de certains théoriciens et praticiens pour l'histoire les conduit à ne plus penser l'architecture seulement comme une quête du nouveau, indifférente à la production passée, mais comme une activité qui prolonge l'œuvre de plusieurs siècles.

L'idée de continuité, historique et formelle de la ville, inscrit alors la construction dans un rapport contextuel incontournable.

Cette nouvelle manière de voir intervient au moment où le Mouvement moderne qui a pensé l'architecture comme une rupture perd de son hégémonie. Pour le Corbusier, par exemple, l'architecture était "hors sol" - sur pilotis - comme un paquebot sur l'océan, étendue indifférente et indifférenciée, et surtout pas dans le port (ill. 1).

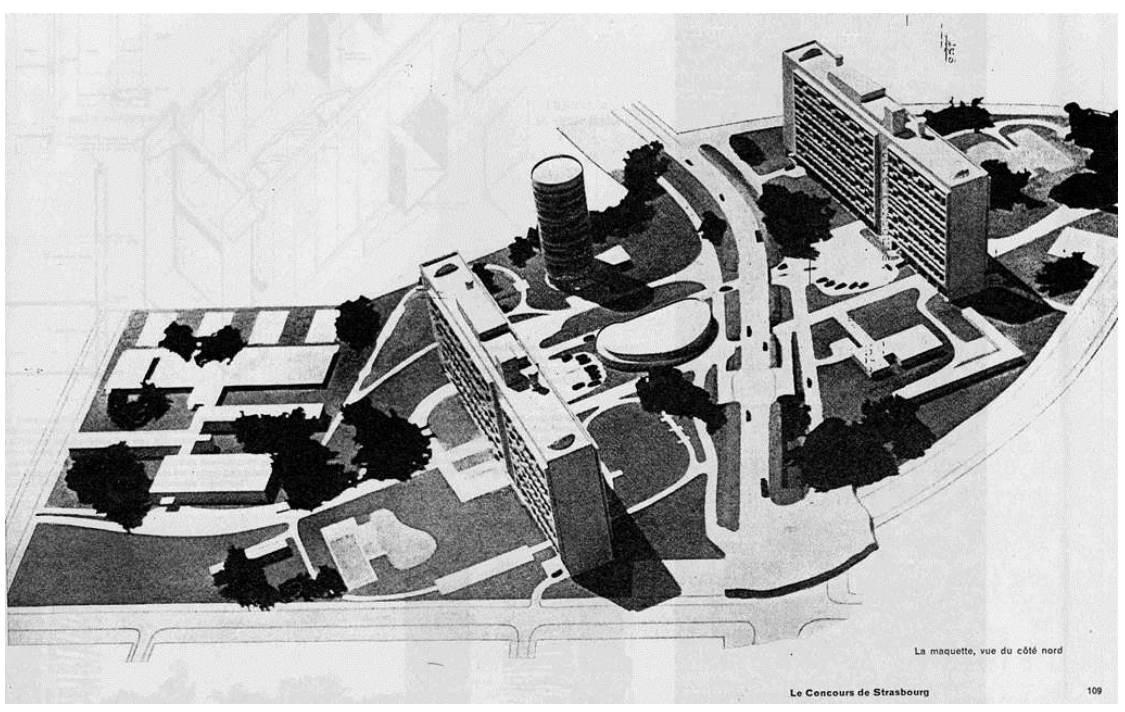

En critiquant ce modèle dès 1966, un architecte comme Aldo Rossi réintroduit l'architecture dans son contexte, celui d'un "paysage" existant, celui de la ville historique. Il exprime une sensibilité qui ne cesse de s'amplifier depuis la loi Malraux de 1962 pour la sauvegarde des centres anciens $^{5}$, et met en avant le concept d'intégration (sous-entendu "au paysage") ${ }^{6}$. Depuis cette loi, on ne s'arrêterait pas de citer, dans le champ de pratique de l'urbanisme, les initiatives, lois et décrets, qui ont institué le respect du paysage ${ }^{7}$.

Aujourd'hui la ville constitue donc un paysage (urbain) et les unités immobilières constitutives de la ville sont considérées en regard de leur contribution à la qualité (cohérence, harmonie, unité) de ce paysage ${ }^{8}$.

\footnotetext{
4. Vernant (Jean-Pierre), "Espace et organisation politique dans la Grèce ancienne" in ESC 20, Paris, 1965, p. 576-595, repris dans La Grèce ancienne, 2. L'espace et le temps, Le Seuil, Points-essais, 1991.

5 . L'édifice monumental protégé n'est plus considéré isolément, mais au coeur d'une architecture mineure qui l'accompagne et mérite également la protection.

6. cf. la pratique française des CAUE (Conseils d'architecture, d'urbanisme et d'environnement, institués par la loi de 1977 sur l'architecture) .

7. Avec les termes plus ou moins affreux propres à l'administration urbanistique tel que ZPPAUP (Zone de Protection du Patrimoine Architectural, Urbain et Paysager, le P de "paysager" étant apparu plus tardivement).
} 


\section{Un nouveau regard sur le grand ensemble}

Le grand ensemble est au centre de cette évolution du regard et par certains côtés, il en est aussi la victime.

Caricature du Mouvement moderne, qui a conçu une Charte dite d'Athènes malgré tout bien plus intéressante (en dépit de son influence certaine sur les grands ensembles), les grands ensembles sont aujourd'hui communément perçus comme un anti-paysage 9 .

En réalité, le grand ensemble est situé à la rencontre de plusieurs logiques. Il est pris aujourd'hui dans une évolution complexe sur laquelle pèsent :

- une situation économique difficile (la crise)

- $\quad$ son rôle spécifique de parc social (HLM pour l'essentiel)

- $\quad$ sa position nouvelle dans le parc d'ensemble du logement (chute vers le bas)

- son vieillissement accéléré comme modèle d'habitation (construction, usage et esthétique confondus)

Je privilégierai ici les aspects qui tiennent à la forme urbaine, mais on notera assez vite qu'ils ne peuvent se réduire à l'apparence physique. Il est en effet difficile de dissocier le paysage de la population qui y habite.

Prenons les choses dans l'ordre, d'abord celui de l'histoire.

\section{L'avènement du confort}

Les grands ensembles, on le sait, sont d'abord une réponse d'urgence à la crise du logement : ils ont apporté un logement décent en termes d'hygiène et de confort à des ménages qui en étaient privés. Tous les témoignages confirment la pertinence temporaire de la réponse.

La société a cependant changé plus vite que le logement et l'élémentaire qu'il apportait s'est révélé rudimentaire, tant comme réponse à la culture d'habiter déjà acquise que comme réceptacle de modes de vie nouveaux. Les couches moyennes ont déserté les HLM pour la maison individuelle, les familles les plus pauvres y ont été rejointes par les familles immigrées regroupées. Comme l'a fort bien dit M. Wievorka, le sas est devenu nasse, le bout de course de la pauvreté.

À la désaffection succède la stigmatisation, et son ampleur, son amplification médiatique, sont à la mesure de la visibilité physique qu'a le grand ensemble dans la ville, par sa taille, par ce que le sens commun appelle son "gigantisme" (ill. 2).

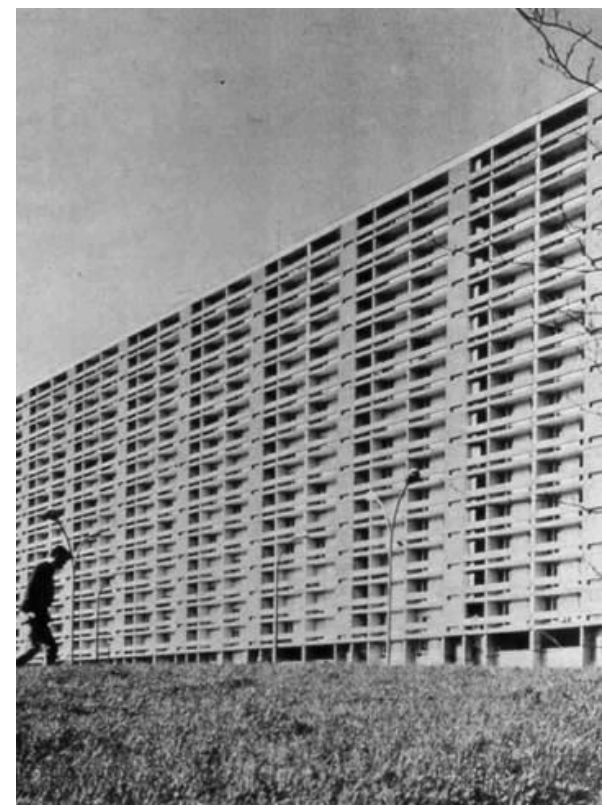

8. En faisant abstraction des architectes qui travaillent à la protection patrimoniale et paysagère (bâtiments classés, secteurs sauvegardés, ZPPAUP), deux situations et attitudes se rencontrent chez ceux qui construisent dans un contexte urbain systématiquement pris en considération :

- celle qui s'efface devant ou se glisse dans le paysage urbain existant (l'Institut du monde arabe dû à l'architecte Jean Nouvel et à l'agence Architecture Studio)

- celle qui recrée le paysage urbain existant (la Bibliothèque F. Mitterrand due à l'architecte Dominique Perrault)

9 . Il semble inspirer le même genre de sentiments que les cimetières de rebuts industriels (casses automobiles ou supertankers à l'abandon dans les fjords nordiques). 
Trente ans plus tard le regard est donc renversé

Changement des représentations, du regard paysagiste? Certes, mais d'abord sur un socle d'évolutions socio-économiques et culturelles considérables au plan des modes de vie et des représentations mentales ${ }^{10}$.

Toutes ces déterminations pèsent donc sur le vécu du grand ensemble, mais pas seulement parce qu'il est grand ensemble et logement social. Pour cette raison aussi qu'il est la fraction du parc de logement la plus dépréciée en regard des canons qui caractérisent la post-modernité.

Cette dépréciation est en effet largement liée au retour qu'a effectuée la société, et surtout cette nébuleuse (contestée) des classes moyennes, vers des valeurs autrefois dominées et écrasées par le mythe d'une modernité absolue, entièrement tournée vers le futur. La post-modernité est désormais plus composite, à la fois ouverte aux technologies de l'information et en même temps plus attentive à certaines effets néfastes du progrès sur les milieux de vie ${ }^{11}$.

Les grands ensembles semblent faire partie aujourd'hui des "dégâts du progrès", et le retournement du regard porté sur eux interroge la qualité de ces lieux. Car à la misère des gens s'ajoute aussi la misère des lieux : l'une et l'autre se renforcent. Sans tomber dans un déterminisme spatial révolu et un misérabilisme social déplacé, et quoiqu'en pensent certains, les lieux ne sont jamais totalement neutres, ils ont toujours un effet social qui renforce ou accompagne d'autres dimensions plus déterminantes.

Le grand ensemble est bien selon moi et pour reprendre l'expression de Marc Augé un "non-lieu", un paysage pauvre, en soi et pour soi d'abord, et pour ses habitants, qui trouvent souvent en dehors de lui les paysages fécondés par leur imagination, comme les jardins familiaux ${ }^{12}$.

Pour tenter de donner un peu de chair à cette analyse de la pauvreté du grand ensemble comme non-lieu, sur laquelle, a priori, il n'y a rien à dire, on peut parler de deux situations propres au grand ensemble et qui rendent compte :

1) de la rationalité techno-administrative et architecturale du grand ensemble, rationalité qu'il a véhiculé comme projet

2) des logiques anthropologiques structurantes qu'il a oubliées comme espace réel, vu et vécu, à différentes échelles de perception.

\section{Premier temps : tableaux d'une exposition}

(le grand ensemble comme pur artefact, gigantesque machine à habiter)

Comme projet, le grand ensemble est une anticipation, une représentation d'un avenir de la ville moderne. On peut discerner deux types de rationalités en œuvre dans cette entreprise :

\footnotetext{
10. Il faut les mentionner pour mémoire :

- des modes de vies transformés par la société fordiste (condition et emploi des femmes, accès à la consommation de masse, automobile et maison, appel de la nature...),

- des comportements qui mettent l'individu (plus maître de ses décisions et en même temps plus seul face à ses choix) au centre de la société,

- une crise du travail qui divise la société en des catégories nouvelles (protégés et précarisés, les "classes multipliées" comme les appelle F. Dubet),

- la transformation d'une immigration de main d'œuvre en une immigration de peuplement qui multiculturalise les classes pauvres.

11. C'est en ce sens que le paysage, qui participe de la construction de ces "lieux de mémoires" chers à Pierre Nora, tempère les risques technologiques.

12. cf. les travaux de Guy Roustang et Daniel Cérézuelle.
} 
- d'abord la rationalité techno-bureaucratique, celle du politique éclairé par les grands commis d'État : le grand ensemble est une solution technique rationnelle qui permet de faire face à la crise du logement.

Cette solution passe

- par le recours à l'industrialisation,

- la restructuration de l'industrie dispersée du bâtiment,

- la création un appareil producteur de normes (le CSTB, 1951),

- la mobilisation de grandes emprises foncières (moyenne $50 \mathrm{ha}$ ), au moyen des décrets de ZUP (nous en verrons plus loin l'effet sur le paysage).

- ensuite les rationalités plus ambigu' de l'architecture, qui vont croiser les anciennes oppositions des anciens et des modernes (celles de Le Corbusier et d'Eugène Baudouin, celles de l'avant-garde et de l'Académie) :

Le grand ensemble est pour ces frères ennemis

- une représentation de la ville moderne, celle de l'ère "machiniste"13, de la standardisation, de la répétition,

- c'est aussi une grande composition moderne, qui se dessine sur le calque comme un tableau (ill. 3)

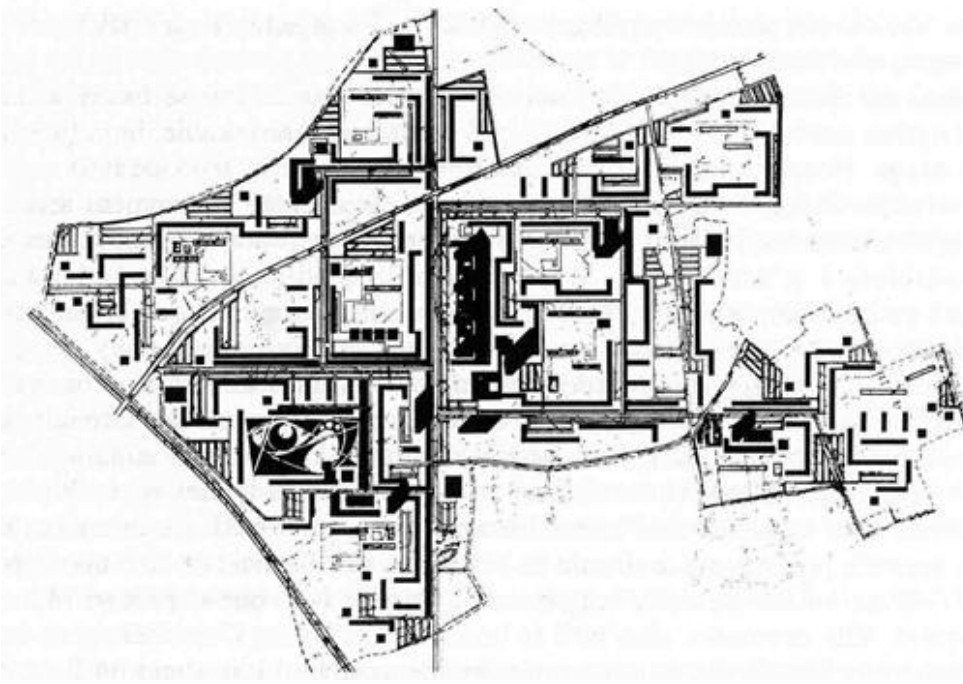

En fait la composition des Beaux-Arts se réapproprie très bien l'esthétique des modernes, en dépit des querelles de l'entre-deux guerres (sur le décor et le toit plat...)

On trouve ainsi dans le grand ensemble le système d'axes de composition, les effets de perspective : la tour au terme de l'axe..., très prisés des académistes (l'axialité est simplement un peu déportée)

. au centre de la composition, les grandes tours projettent de grandes ombres diagonales et symbolisent l'élan et la grandeur de l'opération, sa modernité

. au pourtour, les "grecques" ( I T L ) des barres limitent la ville nouvelle et l'imposent à son environnement.

L'architecte dessine donc le projet de ZUP comme on faisait un projet de grand bâtiment public pour le prix de Rome, pour lui-même, en ignorant le reste de la ville. La ZUP refait le paysage : elle est un tableau avant d'être un ensemble habité. Elle reste une représentation picturale, un exercice académique pour un ensemble à réaliser d'une dimension peu familière. C'est tout à fait le cas, par exemple, pour la ZUP de Pissevin-Valdegour à Nîmes, dessinée par Arsène Henri, Prix de Rome.

Second temps : la ZUP comme paysage vu et vécu

Examinons maintenant le grand ensemble comme réalité construite, à différentes échelles d'appréhension de son paysage : celle de la ville, celle du quartier, celle de l'immeuble et procédons ici à sa lecture anthropo-architecturale.

13. C'est l' "esthétique de l'ingénieur", le modèle de la "machine à habiter", chers à Le Corbusier. 
Nous nous efforcerons de ne dissocier, dans ce travail d'analyse, ni les perceptions interne et externe, ni le paysage réel du paysage représenté, et nous tiendrons compte des interactions qui construisent ces différents niveaux.

A/ L'échelle de la ville

L'impact des grands ensembles sur le cadre physique existant est toujours massif, imposant et brutal. La configuration particulière de la ville peut encore accroitre leur visibilité (c'est le cas, par exemple, à Marseille et à Nîmes) :

- la puissance publique est en effet allée chercher des réserves foncières massives, là où elles pouvaient être trouvées, dans l'environnement pavillonnaire comme sur les belvédères des anciennes "folies" (ill. 4).

On observe en effet des situations différentes : ces réserves sont acquises : tantôt dans l'entre-villes, aux confins des zones pavillonnaires, qu'on ne se gêne pas à l'occasion d'exproprier,

. tantôt sur de grandes propriétés rachetées (à Marseille comme à Nantes).

Ainsi les grands ensembles se donnent à voir par eux-mêmes et par leur situation, dominant de la hauteur de leurs 10/15 étages les pavillons voisins ou

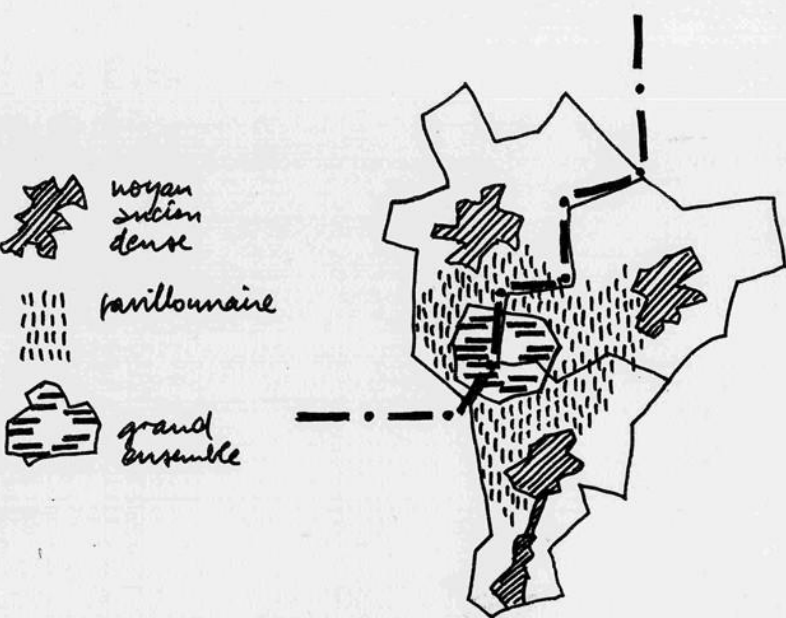

1. Grand ansemble ì cheral su deus déloutements et tors connunus. culminant en gratte-ciel sur les crêtes surplombant les noyaux villageois.

On a là tous les ingrédients qui fondent en un espace et en un temps terriblement concentré une "œuvre" qui s'impose, comme don de l'État-logeur. Cette œuvre doit faire oublier les centres urbains anachroniques et taudifiés, plus ou moins ruinés par la guerre, et les banlieues de mal lotis, alors stigmatisés par les milieux de l'urbanisme et de l'architecture.

Grand' "œuvre" de la modernité, le grand ensemble contraste tant par le paysage nouveau qu'il produit que par son mode de production industriel et son mode de peuplement, centro-administré.

Corps physique étrange et étranger, le grand ensemble y reçoit aussi les corps d'une population "parachutée" qui ne résulte pas du processus d'agglutinement progressif qui caractérise la ville ancienne.

Si l'on pense le paysage comme une association d'ambiances physiques et populationnelles, on ne peut abstraire l'effet de double étrangeté, physique et humaine que produit le grand ensemble.

B/ l'échelle du quartier

Ensemble moderne dont les hérauts affirment d'emblée la mission urbaine de remise en ordre contre l'anarchie pavillonnaire et l'insalubrité intraurbaine, le grand ensemble provoque aussi comme paysage urbain construit, à la fois visuellement et symboliquement, une inversion des valeurs urbaines (ill. 5). 


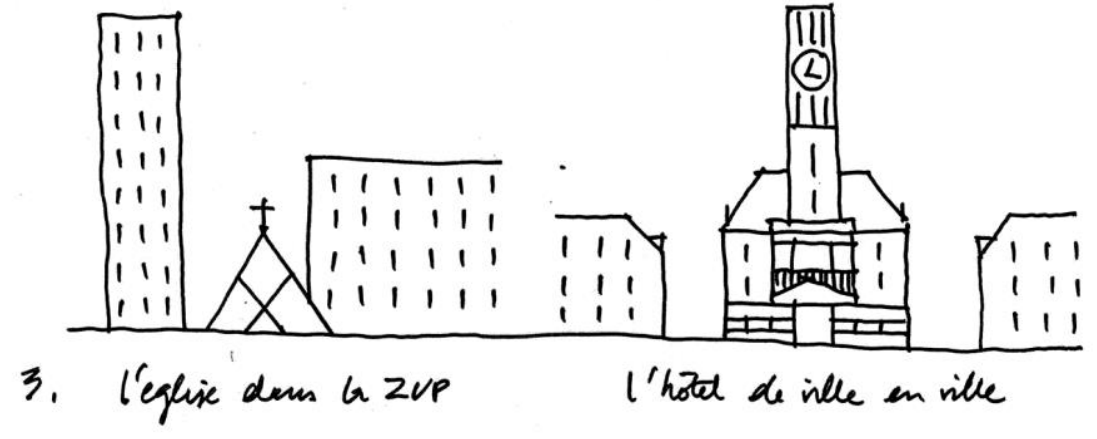

Celles-ci ordonnent historiquement la taille des constructions en fonction de leurs rôles politique et symbolique. Ainsi la hauteur des immeubles d'habitation est généralement subordonnée à celle des édifices publics ou communautaires. Cette hiérarchie de dimension, ce rapport d'échelle donnent sens au rapport de l'individu à la société.

Dans la ville classique occidentale, les habitations, disposées en îlots, ont un gabarit (ou un épannelage) de dimension inférieure à celui de l'édifice singulier (comme l'appelle l'architecte italien Aldo Rossi), qu'il s'agisse de l'église paroissiale, de l'hôtel de ville, ou du siège de banque aujourd'hui. Le campanile, le beffroi ou la flèche du clocher réalisent ce contraste, qui se manifeste avec force dans le paysage.

Les grands ensembles ont totalement inversé ce rapport : la tour de logement domine ce qu'on appelle de façon la plus administrative l' "équipement de quartier". La haute tour et la longue barre sont des empilements de logements sociaux dans des immeubles de grande dimension ${ }^{14}$. Le logement s'investit alors d'une monumentalité inhabituelle, tandis que l'édifice destiné à l'association s'efface dans une écrasante banalité.

On n'a sans doute pas assez réfléchi à la signification qu'il y avait à habiter une tour ${ }^{15}$, d'une part, et sur le sens que pouvait suggérer au regard d'autrui un appartement noyé dans l'immensité répétitive des fenêtres des tours ou des barres. Le logement porte en effet une valeur domestique marquée par la privauté (home, sweet home) qui s'accorde difficilement à l'échelle monumentale d'un immeuble de grande hauteur. La hauteur de la tour signifie en effet bien plus "l'entreprise publique du logement" (comme dit Henri Raymond) que l'initiative de l'habitant.

La tour comme la barre, en présentant ce désaccord parfait ${ }^{16}$ entre l'intérieur domestique et l'extérieur industriel-étatique, véhicule un processus de désidentification de l'habitant que ce dernier traduit souvent dans son propre langage par le terme d' "anonymat". Comme traduction de l'œuvre grandiose de l'Etat-providence, elle projette sur l'habitant une image d'assisté qui se traduit dans certains comportements de honte, lorsqu'il s'agit de dire à autrui où l'on habite, et a fortiori lorsqu'il s'agira d'aller jusqu'à l'invitation : le grand ensemble est devenu un paysage matériel qui produit rétroactivement une représentation de soi et pour les autres négative.

C/ l'échelle de l'immeuble

L'architecture de l'habitation occidentale est historiquement marquée par son orientation. Il s'agit moins ici de l'orientation héliothermique qui parachève chez les architectes

\footnotetext{
14. Certes, la jouissance des logements supérieurs peut toujours présenter un charme et un privilège inhabituel (à condition que l'ascenseur ne soit pas en panne), et comme à Marseille l'avantage d'une vue superbe sur la Méditerranée.

15. Edward T. hall s'en est alarmé pour la population noire dans La dimension cachée, p. 205.

16. Je force le sens de ce qu'Henri Raymond appelle la "relation imparfaite" entre l'intérieur et l'extérieur.
} 
modernes l'hygiénisme du XIXe siècle, que l'orientation topologique et symbolique qui structure l'architecture et la distingue en devant/derrière, public/privé17.

La mise en parallèle un peu caricaturale présentée dans les illustrations 6 et 7 nous aidera à représenter la manière dont cette dimension symbolique a pu disparaître dans la signification portée par la barre et la tour du grand ensemble. Ce schéma s'efforce de mettre en évidence la perte, dans le grand ensemble, de certains traits morphologiques fondamentaux. Ils ont à mon sens ont une valeur anthropologique essentielle, qui nous rappelle l'apparentement de l'habitation au corps (troisième peau après l'habit).

Le grand ensemble fait ainsi l'économie de dispositifs formels marquant des orientations et des hiérarchies qui, alors, font défaut à l'accomplissement des conduites corporelles et des pratiques de l'espace propres aux habitants ou à ceux qui ont une relation avec eux.

. au plan horizontal, de l'entrée vers le fond de la maison (ill. 6)

Le HLM perd ce qui, dans la maison et l'immeuble haussmannien, ordonnait la relation du domicile avec l'espace public : la distinction d'un devant et d'un derrière, d'une façade principale qui se donne à voir et d'un jardin privatif plus caché ou d'une cour de service où sont refoulées des choses moins

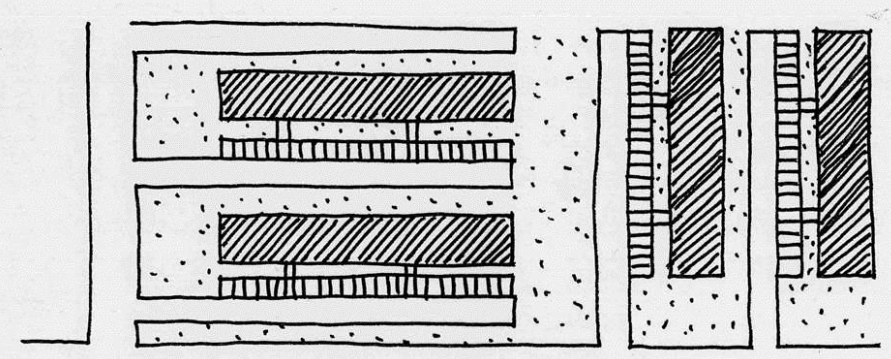

2- la confunoir du devant ot de devriér. exposables de la vie domestique.

Cette structuration du présentable et de l'imprésentable (du montré et du caché, du propre et du sale, du beau et du laid, ou du public et de l'intime), disparaît dans l'indifférenciation des faces de la barre ou de la tour.

Dans le même sens la voirie dessert les entrées, et les parkings représentent encore sur l'arrière le stationnement des mêmes automobiles : le derrière est donc un second devant, et ce qui voudrait se cacher y parvient difficilement.

Ces immeubles-objets sont désormais inorientés (la notion de façade principale disparaît). Ils ont perdu ce rapport anthropomorphique signifiant qu'entretenait initialement la maison avec le corps humain, lui-même orienté (la façade n'est pas sans évoquer la face).

. au plan vertical, de la base au sommet, d'une entrée à l'autre (ill. 7)

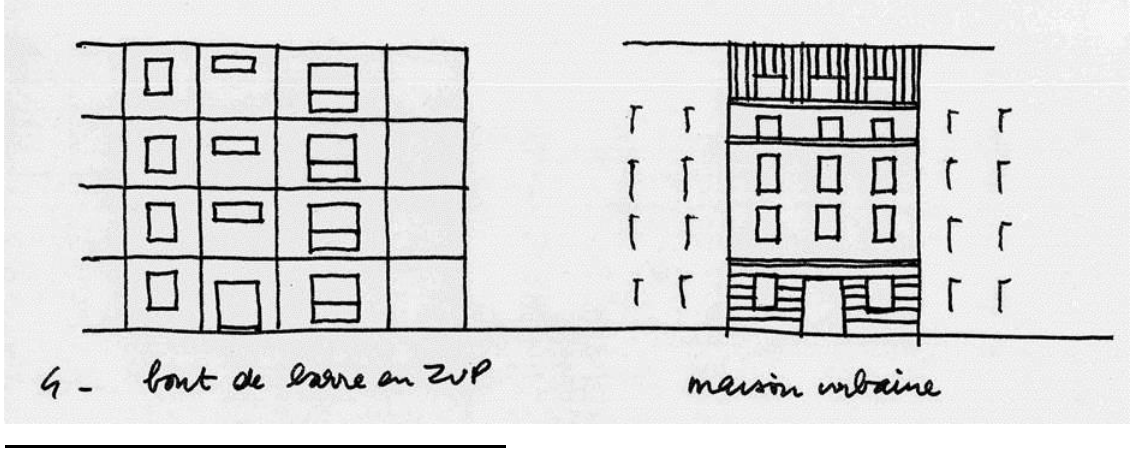

La division parcellaire de la ville ancienne associe des types reconnus, voisins dans leurs configuration sans être totalement identiques : ils donnent l'image

17. C'est le mérite de l'équipe Raymond-Haumont de l'avoir souligné pour les pavillons et Pierre Bourdieu pour la maison Kabyle (annexe à Le sens pratique, Minuit, Paris, 1981). Bachelard et Lévi-Strauss les avaient précédés, le premier avec sa maison tierce (de la cave au grenier, du dyonisiaque à l'appolinien), le second avec la division de son village Bororo et l'orientation cosmique des fratries. 
d'une société à l'échelle du quartier qui partage des valeurs sans que la diversité des familles et des individus qui la composent soit, comme dans le grand ensemble, réduite à l' "identiquité" par le dépouillement qu'expriment la répétition des tours et des barres, et, dans cette répétition, celle plus standardisée encore des panneaux de façades qui les composent.

De droite à gauche, de bas en haut, l'empilement des "cellules" redit cette "ration"18 de logement qu'est le logement de masse administrativement attribué des années 1950, alors que la plus modeste baraque des mal lotis portait l'infime message d'une identité toujours digne dans son dénuement ${ }^{19}$.

Autre aspect : une maison est habituellement fondée, d'une manière ou d'une autre, et la base de la construction était autrefois réalisée dans un matériau plus résistant qui exprimait, selon la métaphore végétale, l'enracinement de l'immeuble. À la fondation s'opposait la "couverture", dont la charpente dit encore le rapport au lacis des branches qui portent la frondaison. D'une certaine manière la verticalité de notre condition humaine se dit dans l'élévation de la maison.

Ce reste d'analogie anthropomorphique disparaît dans la plupart des immeubles de grands ensembles pour céder la place à la rationalité obsédante, industrielle et productive, homogène et quadrillée, du standard de la façade HLM années 1960.

Au total, l'addition des pertes de sens est lourde : elle somme une série de simplifications formelles qui délestent le cadre bâti, dans toutes ses dimensions, d'une série de dispositifs qui le mettent en rapport concret avec un site, pour en faire un territoire. Or c'est précisément en s'inscrivant dans un lieu, qui par sa potentialité d'accueil fait déjà sens, qu'un nouvel établissement humain, est capable, par l'attention au lieu que porte son architecture, de redonner sens à ce territoire.

\section{En guise de conclusion}

Un débat inépuisable a depuis longtemps opposé les négateurs absolus des effets de la forme urbaine sur la vie sociale et ceux qui y plaçaient un déterminisme excessif. Une approche culturelle des objets techniques (ceux que M. Serres appelle des quasi-objets et que B. Latour nomme des hybrides) me paraît permettre de dépasser cette opposition réductrice.

On peut, en ce sens, analyser le grand ensemble comme un paysage, d'abord comme artefact picturo-architectural, ensuite réifié dans l'espace socio-spatial de la ville, perçu enfin comme segment d'un environnement urbain plus vaste, au sein duquel il prend une valeur relative. D'abord positivement perçu dans l'offensive de la modernité triomphante des trente glorieuses, il est aujourd'hui réévalué en négatif et, il ne faut pas le cacher, la présence des familles dites "lourdes" pèsent sur cette réévaluation négative.

On pourrait penser qu'à l'exemple des centres anciens dégradés, les grands ensembles retrouvent une habitabilité nouvelle. Il semble cependant que la désaffection du grand ensemble tienne à des défauts structurels plus décisifs : ils ne renvoient pas tant à leur caractéristiques techniques, qu'à une configuration typologique et morphologique déficiente. Elle nie certains universaux de l'habité et de l'urbanité, que nous avons tenté de cerner dans cette approche anthropo-architecturale du grand ensemble.

Pressés par la désertion massive de certains de ces ensembles (qui ne sont pas seulement frappés par une vacance technique, mais aussi par le refus d'y être logés des plus

18. Expression de l'architecte allemand Ernst May, figure importante du Mouvement moderne.

19. voir à ce sujet Colette Pétonnet, On est tous dans le brouillard, Galilée, Paris, 1979. 
pauvres), désertion dont le coût est très important, les organismes HLM ont entamé avec l'État une entreprise à long terme de résorption des grands ensembles ${ }^{20}$.

Axée sur la reconstruction-démolition, cette perspective laisse apparaître que la réhabilitation inspirée de la démarche en centre ancien est inopérante sur les grands ensembles, sans doute parce que ces quartiers ne portent pas ces qualités paysagères appréciées des contemporains, et, selon moi, aussi, ces traits structurels et symboliques propres à la culture urbaine occidentale qui permettent de surmonter l'obsolescence relative des immeubles anciens.

\section{BIBLIOGRAPHIE SOMMAIRE}

AUGÉ, Marc (1992), Non-lieux, introduction à une anthropologie de la surmodernité, Le Seuil, Paris.

BÉGUIN, François (1995), Le paysage, Flammarion, Paris.

CHOAY, Françoise (1981), La règle et le modèle, Le Seuil, Paris.

DUBET, François (1998), Dans quelle société vivons-nous?, Le Seuil, Paris.

HALL, Edward T. (1971), La dimension cachée, Le Seuil, Paris (édition américaine, 1966).

LUGINBUHL, Yves (1989), Paysages, La Manufacture, Paris.

PÉTONNET, Colette (1979), On est tous dans le brouillard, Galilée, Paris.

PINSON, Daniel (1996), Architecture et modernité, Flammarion, Paris.

PINSON, Daniel (1995), "La monumentalisation du logement," in Les cahiers de la recherche architecturales, $\mathrm{n}^{\circ}$ 38-39, Banlieues, Parenthèses, Marseille.

PINSON, Daniel (1987), Voyage au bout de la ville, histoires, gens et décors de la ZUP, ACL-Crocus, Saint-Sébastien-sur-Loire.

RAYMOND, Henri (1984), L'architecture, les aventures spatiales de la raison, CCI-Centre Pompidou, Paris.

ROSSI, Aldo (1981), L'architecture de la ville, L'Équerre, Paris (édition italienne, 1966).

SERRES, Michel (1992), Éclaircissements, entretiens avec B. Latour, F. Bourin, Paris (réédition coll. Champs, Flammarion, 1994).

SITTE, Camillo (1980), L'Art de bâtir les villes, L'Équerre, Paris (édition allemande, 1889).

UNWIN, Raymond (1981), L'étude pratique des plans de villes, L'Équerre, Paris (édition anglaise, 1909).

TURNER, John F.C. (1979), Le logement est votre affaire, Le Seuil, Paris (édition américaine, 1971).

\section{LISTE DES ILLUSTRATIONS}

Ill. 1. Les Unités d'Habitation de Grandeur Conforme proposées par Le Corbusier au Concours de Strasbourg, 1951 (Euvre complète).

Ill. 2. Anonymat (photo parue dans Le Monde dans les années 1980)

Ill. 3. Plan de masse initial de la ZUP Bellevue (1957, Architecte : Marcel Favraud)

Ill. 4. Principes d'implantation des grands ensembles dans une métropole à banlieues (dessin de l'auteur)

Ill. 5. L'inversion des valeurs urbaines (dessin de l'auteur)

Ill. 6. La perte du devant-derrière (dessin de l'auteur)

Ill. 7. La perte du haut-bas, du mitoyen (dessin de l'auteur)

20. Pinson (Daniel), "La résorption progressive des grands ensembles en France : enjeux qualitatifs et sociaux", communication au Colloque de l'APERAU: "Les évolutions des politiques du logement et leurs incidences sur la planification urbaine", Tunis, 1998. 